\title{
THE DISTANT DRAGNS SURVEY
}

\author{
J. D. B. LAW-GREEN \\ University of Manchester, \\ NRAL Jodrell Bank, Cheshire SK11 9DH, UK
}

\section{Introduction}

DRAGNs (Double Radio sources Associated with Galactic Nuclei, Leahy 1991) are the class of powerful extragalactic radio sources thought to be produced by the interaction of a jet with the ambient medium. They exhibit strong cosmological evolution in comoving number density; at $z \sim 2$ the "classical double" FR II DRAGNs were $\sim 1000$ times as common as they are now (Dunlop \& Peacock 1990).

To understand this, systematic studies of complete DRAGN samples at low and high $z$ and differing levels of flux density are required, in order to resolve the $P-z$ ambiguity. The Distant DRAGNs Survey is a longterm project to image with the VLA and MERLIN, matched samples of DRAGNs at high redshift.

\section{Observations}

The DDS sources are drawn from three optically almost complete lowfrequency samples: 18 DRAGNs from 3CR (Spinrad 1985) at $z>1.5$ and 23 from the overlapping "6C/B2 2-Jy" samples (Allington-Smith 1982, Eales $1985)$ at $z>1.7$. Each source is observed at two frequencies from $408 \mathrm{MHz}$, $1.4 \mathrm{GHz}$ and $5 \mathrm{GHz}$ using MERLIN or VLA. Sub-kpc linear resolution is achieved at $1.4 \mathrm{GHz}$ and higher, probing similar scales to HST imaging. Full polarimetry is obtained at $1.4 \mathrm{GHz}$ and above.

The observations are currently $\sim 60 \%$ complete. The project has "longterm" approval status from the MERLIN TAC.

\section{Results}

Interim analysis of the images obtained so far reveals several trends:- 

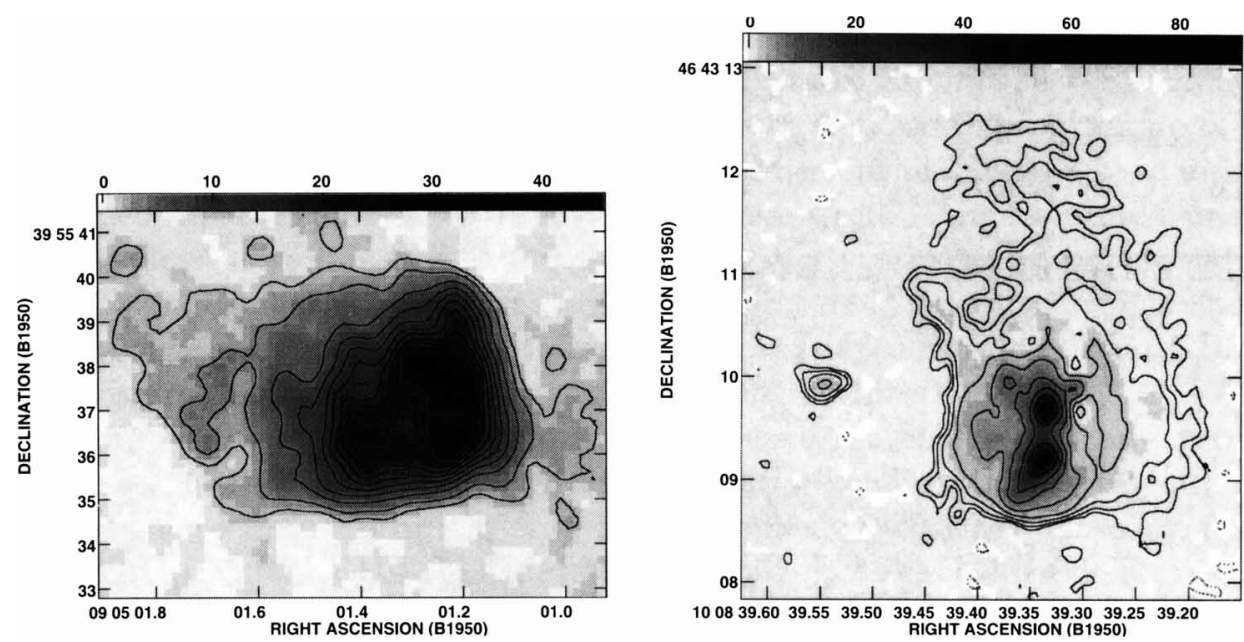

Figure 1. Suspected lensing in distant DRAGNs: (a) "Quad" in western lobe of $4 \mathrm{C39.24}$ (MERLIN $408 \mathrm{MHz}$; Law-Green et al. 1995). (b) Eastern lobe of 3C 239 with lensed counterimage (MERLIN+VLA 1.4/1.6 GHz MFS; Law-Green et al., in prep.). An $R=21.8$ foreground galaxy lies very close to the counterimage.

Lensing: Following the method of Kochanek \& Lawrence (1990) the expected number of gravitationally lensed sources in our sample is 0.30 in a complete set of MERLIN $1.4 \mathrm{GHz}$ images. We find at least two possible lenses (Figure 1) supporting the idea that distant radio sources are strongly amplified by lensing (Hammer \& LeFevre 1989).

Frontflows: Very steep-spectrum $(\alpha>2)$ plumes beyond the radio hotspots seem common in distant DRAGNs, possibly relics of previous outbursts.

Giant: 4C39.24 was found during the DDS survey to be the most distant known giant radio galaxy (Law-Green et al. 1995). The source is $111^{\prime \prime}$ across at a redshift of $z=1.883$, equivalent to $D=690 \mathrm{kpc}\left(\Omega_{0}=0.2\right)$.

Compactness: The compactness (fraction of flux in compact hotspots) of distant sources appears greater in less luminous DRAGNs.

\section{References}

Allington-Smith J. R., 1982, MNRAS, 199, 611

Dunlop J. S., Peacock J. A., 1990, MNRAS, 247, 19

Eales S. A., 1985, MNRAS, 217, 149

Hammer F., LeFevre O., 1989, ApJ, 357, 88

Kochanek C. S., Lawrence C. R., 1990, $A J, 99,1700$

Law-Green J. D. B., Eales S. A., Leahy J. P., Rawlings S. G., Lacy M., 1995, MNRAS, in press

Leahy J. P., 1991, in Röser H.-J., Meisenheimer K., eds. Jets in Extragalactic Radio Sources, Springer-Verlag, Berlin, p.1 